\title{
Influence of correlated visual cues on auditory signal detection'
}

\author{
R. A. KINCHLA, NEW YORK UNIVERSITY \\ J. TOWNSEND, STANFORD UNIVERSITY \\ J. I. YELLOTT, JR., UNIVERSITY OF MINNESOTA R. C. ATKINSON, STANFORD UNIVERSITY
}

Two experiments investigated the effects on auditory signal detection of introducing visual cues that were partially correlated with the signal events. The results were analyzed in terms of a detection model that assumes that such cue-signal correlations will not affect sensitivity, but will instead cause the subject to develop separate response biases for each cue. The model specifies a functional relationship between the asymptotic values of these cuecontingent biases. The overall results of the experiments supported the detection assumptions of the model and the general bias learning assumption, but indicated a more complex learning process than that specified by the model.

Several current models for signal detection represent performance as jointly determined by psychophysical variables (e.g., signal parameters) and such "background" variables as relative frequency of various signal events on previous trials (Atkinson, Carterette, \& Kinchla, 1962; Luce, 1963; Swets, Tanner, \& Birdsall, 1961). Typically these background variables determine the response bias parameters of a hypothetical decision process relating signal-produced sensory states to overt responses. To the extent that the subject's choice is controlled by these biases, rather than by discriminative information provided by the signal presentation, there is a formal similarity between detection experiments and probability learning experiments (Atkinson, Bower, \& Crothers, 1965, Ch. 5). The present study deals with a detection situation analogous to probabilistic discrimination learning. In a probabilistic discrimination learning experiment each trial is initiated by one of a set of cues, each of which corresponds to a particular probability distribution over the set of possible trial outcomes. The comparable detection situation is called a cued detection task. Here each detection trial is initiated by one of a set of cues, and each cue corresponds to a distinct probability distribution over the possible signal events. (That is, whenever a trial is initiated by cue $c_{i}$ the probability of signal event $S_{i}$ is given by $\gamma_{i j}$ ) If these distributions are different the cues may be said to be correlated with the signal events. The results of probabilistic discrimination learning experiments (Popper \& Atkinson, 1958; Atkinson, Bogartz, \& Turner, 1959) suggest that correlated cues should come to control behavior in a cued detection task; i.e., a subject will come to hold several response biases simultaneously, with the effective bias on a given trial being determined by the cue on that trial.

The possibility of multiple-response biases was in- vestigated in the context of an auditory two-interval forced-choice detection task involving three visual cues. The results were analyzed in terms of an extension of a detection model developed by Atkinson \& Kinchla (1965) and Luce (1963). For present purposes this model can be outlined as follows. Each trial of the experiment is initiated by one of three cues: $c_{1}, c_{2}$, or $c_{3}$. The experimenter then presents the signal in interval 1 or interval 2. It is assumed that with probability $\sigma$ the occurrence of a signal in interval $i$ gives rise to an "unambiguous" sensory state $s_{i}$, whereas with probability $1-\sigma$ an ambiguous sensory state occurs. The ambiguous sensory state is denoted by $b_{h}$ when the cue is $c_{h}$. If the stimulus presentation elicits $s_{i}$ the subject makes response $A_{i}$ indicating that he thought the signal was in interval $i$. If the ambiguous sensory state $b_{h}$ occurs the subject makes response $A_{1}$ with probability $P_{h, n}$, where $n$ is the trial index. The $P_{h, n}$ can be thought of as momentary response bias parameters associated with the sensory states $b_{h}$, whereas $\sigma$ is a measure of the subject's sensitivity to the signal.

The following notation is used to refer to the various events that occur on trial $n$ :

$$
\begin{aligned}
& C_{h, n} \text { : the occurrence of cue } C_{h}(h=1,2,3) ; \\
& S_{i, n} \text { : presentation of the signal in interval } i(i=1,2) \text {; } \\
& A_{i, n} \text { : the occurrence of response } A_{j}(i=1,2) ; \\
& E_{k, n} \text { : presentation of information feedback indicating that the } \\
& \text { correct response was } A_{k}(k=1,2) \text {. }
\end{aligned}
$$

In our experiments correct information feedback was given to the subject on every trial, i.e., $E_{1}$ always occurred on an $S_{1}$ trial, and $E_{2}$ on an $S_{2}$ trial. The relationship between the cues and the signal events is summarized by the conditional probabilities:

$$
\gamma_{h}=\operatorname{Pr}\left(S_{1, n} \mid C_{h, n}\right), \quad(h=1,2,3) .
$$

If $\gamma_{1}=\gamma_{2}=\gamma_{3}$ the cues are uncorrelated with the signal events, otherwise the schedule is said to be cue dependent or correlated. The probability of cue $C_{h}$ on any trial is denoted by $\lambda_{h}(h=1,2,3)$.

From the assumptions of the model it follows that

$$
\begin{aligned}
& \operatorname{Pr}\left(\mathrm{A}_{1, n} \mid \mathrm{S}_{1, n} \mathrm{C}_{h, n}\right)=\sigma+(1-\sigma) \mathrm{E}\left(\mathrm{P}_{\mathrm{h}, \mathrm{n}}\right) \\
& \operatorname{Pr}\left(\mathrm{A}_{1, n} \mid \mathrm{S}_{2, n} \mathrm{C}_{h, n}\right)=(1-\sigma) \mathrm{E}\left(\mathrm{P}_{\mathrm{h}, \mathrm{n}}\right),
\end{aligned}
$$

where $E\left(p_{h, n}\right)$ denotes the expectation of $P_{h, n}$. These equations in turn imply 


$$
\begin{aligned}
\operatorname{Pr}\left(A_{1, n} \mid S_{1, n} C_{h, n}\right) & =\operatorname{Pr}\left(A_{1, n} \mid S_{2, n} C_{h, n}\right)+\sigma \\
E\left(p_{h, n}\right) & =\frac{\operatorname{Pr}\left(A_{1, n} \mid S_{2, n} C_{h, n}\right)}{1-\sigma}
\end{aligned}
$$

For the two-interval forced-choice situation the receiver-operating-characteristic (ROC) curve is a plot of the observed proportion of "hits" $\hat{P}_{r}\left(A_{1} \mid S_{1}\right)$ against the observed proportion of 'false alarms" $\hat{P}_{r}\left(A_{1} \mid S_{2}\right)$. The present model implies that for each value of the bias parameter the point $\left[\operatorname{Pr}\left(A_{1} \mid S_{2}\right), \operatorname{Pr}\left(A_{1} \mid S_{1}\right)\right]$ will fall on a line that has slope one and $\operatorname{Pr}\left(A_{1} \mid S_{1}\right)$ intercept $\sigma$. If a block of $C_{h}$ trials is used to determine a point on the ROC curve the model implies that

$$
E\left[\hat{P}_{r}\left(A_{1} \mid S_{1} C_{h}\right)\right]=\sigma+E\left[\hat{P}_{r}\left(A_{1} \mid S_{2} C_{h}\right)\right]
$$

where $\operatorname{Pr}\left(A_{1} \mid S_{i} C_{h}\right)$ is the observed proportion of $A_{1}$ responses over those $C_{h}$ trials on which the signal event was $S_{i}$. Thus if the ROC curve is formed by plotting the points $\left[\hat{\operatorname{Pr}}\left(A_{1} \mid S_{2} C_{h}\right), \hat{P}_{r}\left(A_{1} \mid S_{1} C_{h}\right)\right]$ for each $C_{h}$, the model predicts that the "expected" ROC curve will be a straight line with slope one and intercept $\sigma$. Moreover it can be shown that under very general assumptions on the $P_{h, n}$ process the difference $\hat{\operatorname{Pr}}\left(A_{1} \mid S_{l} C_{h}\right)-\operatorname{Pr}\left(A_{1} \mid S_{2} C_{h}\right)$ converges in probability to $\sigma$ as the block size increases. Consequently to estimate $\sigma$ we use the consistent estimator

$$
\hat{\sigma}=\hat{\operatorname{Pr}}\left(A_{1} \mid S_{1} C_{h}\right)-\hat{P}_{r}\left(A_{1} \mid S_{2} C_{h}\right) .
$$

Note that this estimator can be used to obtain separate estimates of $\sigma$ for each cue. To estimate the response bias on $C_{h}$ trials, we use the estimator suggested by Eq. 2:

$$
\hat{P}_{h}=\frac{\hat{P}_{r}\left(A_{1} \mid S_{2} C_{h}\right)}{1-\hat{\sigma}}
$$

For purposes of analysis a linear learning model for the response biases will be considered; namely

$$
p_{h, n+1}= \begin{cases}(1-\theta) p_{h, n}+\theta, & \text { if } c_{h, n}, b_{h, n}, s_{l, n} \\ \left(1-\theta^{\prime}\right) p_{h, n} & , \text { if } c_{h, n}, b_{h, n}, s_{2, n} \\ p_{h, n} & \text { otherwise }\end{cases}
$$

where $b_{h, n}$ denotes the occurrence of the ambiguous sensory event $b_{h}$ on trial $n$. This model predicts that

$$
P_{h}=\lim _{n \rightarrow \infty} E\left(p_{h, n}\right)=\frac{\gamma_{h}}{\gamma_{h_{z}}+\left(1-\gamma_{h}\right) \phi}
$$

where $\phi=\theta^{\%} / \theta$. If $\phi=1$, the limiting response bias on $c_{h}$ trials matches the conditional probability of an $s_{1}$ signal event given cue $c_{h}$.

Experiment I investigated the effects of introducing a cue-signal correlation after subjects had had considerable practice on an uncorrelated schedule.

\section{EXPERIMENT I}

\section{Method}

The task employed was the two-interval, forcedchoice detection of a $100 \mathrm{msec}$. $1000 \mathrm{cps}$ signal in a background of band-limited Gaussian noise. The noise was produced by a Grayson-Stadler Model 455-B noise generator, and was presented binaurally to the subject over Permaflux PDR-10 earphones. Each subject sat facing a display on which there were two arrays of lights: a vertical array of three cue lights, and a horizontal array of three interval lights. One of the cue lights came on at the beginning of each trial. One second later the three-interval lights blinked on, one after the other, starting from the left. Each interval light was on for $100 \mathrm{msec}$, and there was a $500 \mathrm{msec}$. off period between the offset of one light and the onset of the next light. The first interval light was an alert signal, while the next two indicated the test intervals. ${ }^{2}$ On every trial the signal tone was added to the background noise during one of the test intervals. The subject's task was to decide which interval contained the signal. He was given $1.7 \mathrm{sec}$. following the second test interval to indicate his choice by pressing a pushbutton located directly under the appropriate interval light. At the conclusion of the response period information feedback was provided by a 1-sec. illumination of the pushbutton corresponding to the correct response. The total time for each trial was 4 sec.; the intertrial delay was 2 sec.

The correspondence between these experimental events and the notation introduced earlier is as follows: the occurrence of a cue light corresponds to $c_{1}, c_{2}$, or $C_{3}$ respectively, beginning with the uppermost cue light. The occurrence of the signal in the first or second test interval corresponds to $S_{1}$ and $S_{2}$ respectively. Similarly, $A_{1}$ denotes a response indicating that the subject believed the signal occurred in the first test interval and $A_{2}$ denotes a response indicating that the signal occurred in the second test interval. The illumination of the $A_{2}$ response button corresponds to the information feedback event $E_{1}$, and illumination of the $A_{2}$ button corresponds to $E_{2}$.

The programming of events during each experimental session, as well as the recording of the data, was fully automated. Program information was automatically read from computer-produced punched paper cards. The trial number, cue light, the interval in which the signal appeared, the subject's response, and his response latency were automatically recorded on similar cards for eventual computer analysis.

A particular noise level was selected for each subject during three days of preliminary testing. During these sessions the three cue lights occurred equally often and were uncorrelated with the signal events. The subjects ran through 360 trials each day with a fixed signal amplitude. The noise amplitude was varied until the experimenter was satisfied that a level had been reached at which the subject would obtain approximately 75 per cent correct responses. The noise was 
Table 1. Daily estimates of response probabilities from Experiment I

\begin{tabular}{|c|c|c|c|c|c|c|c|c|}
\hline Day & $\hat{\operatorname{Pr}}\left(\mathrm{A}_{1} \mid \mathrm{S}_{1} \mathrm{C}_{1}\right)$ & $r\left(A_{1} \mid S_{2} C_{1}\right)$ & $\hat{\operatorname{Pr}}\left(\mathrm{A}_{1} \mid \mathrm{S}_{1} \mathrm{C}_{2}\right)$ & $\hat{F r}\left(A_{1} \mid S_{2} C_{2}\right)$ & $\hat{\operatorname{Pr}}\left(\mathrm{A}_{1} \mid \mathrm{C}_{1}\right)$ & $\hat{\operatorname{Pr}}\left(A_{1} \mid C_{2}\right)$ & $\hat{\operatorname{Pr}}$ (Correct) & $\hat{\operatorname{Pr}}\left(A_{1}\right)$ \\
\hline 1 & 0.837 & 0.287 & 0.849 & 0.321 & 0.560 & 0.587 & 0.770 & 0.574 \\
\hline 2 & 0.746 & 0.331 & 0.758 & 0.579 & 0.541 & 0.567 & 0.698 & 0.555 \\
\hline 3 & 0.764 & 0.412 & 0.765 & 0.411 & 0.587 & 0.587 & 0.676 & 0.587 \\
\hline 4 & 0.679 & 0.328 & 0.720 & 0.379 & 0.503 & 0.550 & 0.673 & 0.527 \\
\hline 5 & 0.713 & 0.293 & 0.735 & 0.325 & 0.503 & 0.525 & 0.710 & 0.514 \\
\hline 6 & 0.676 & 0.285 & 0.683 & 0.282 & 0.479 & 0.483 & 0.698 & 0.481 \\
\hline 7 & 0.737 & 0.328 & 0.758 & 0.325 & 0.532 & 0.542 & 0.710 & 0.537 \\
\hline 8 & 0.743 & 0.326 & 0.692 & 0.321 & 0.528 & 0.507 & 0.699 & 0.518 \\
\hline 9 & 0.781 & 0.231 & 0.782 & 0.315 & 0.506 & 0.548 & 0.754 & 0.527 \\
\hline 10 & 0.720 & 0.252 & 0.721 & 0.249 & 0.604 & 0.367 & 0.736 & 0.485 \\
\hline 11 & 0.756 & 0.278 & 0.733 & 0.279 & 0.636 & 0.393 & 0.736 & 0.515 \\
\hline 12 & 0.793 & 0.335 & 0.736 & 0.247 & 0.676 & 0.368 & 0.756 & 0.523 \\
\hline 13 & 0.805 & 0.290 & 0.704 & 0.255 & 0.676 & 0.366 & 0.758 & 0.523 \\
\hline 14 & 0.753 & 0.274 & 0.673 & 0.234 & 0.631 & 0.345 & 0.743 & 0.486 \\
\hline 15 & 0.795 & 0.249 & 0.647 & 0.236 & 0.661 & 0.336 & 0.760 & 0.499 \\
\hline 16 & 0.806 & 0.244 & 0.736 & 0.199 & 0.666 & 0.332 & 0.789 & 0.499 \\
\hline 17 & 0.815 & 0.300 & 0.756 & 0.195 & 0.687 & 0.336 & 0.789 & 0.511 \\
\hline 18 & 0.825 & 0.263 & 0.732 & 0.168 & 0.680 & 0.310 & 0.804 & 0.495 \\
\hline 19 & 0.791 & 0.348 & 0.690 & 0.211 & 0.666 & 0.346 & 0.758 & 0.507 \\
\hline 20 & 0.769 & 0.282 & 0.725 & 0.221 & 0.540 & 0.460 & 0.753 & 0.500 \\
\hline 21 & 0.780 & 0.248 & 0.716 & 0.220 & 0.514 & 0.470 & 0.757 & 0.492 \\
\hline 22 & 0.735 & 0.258 & 0.742 & 0.217 & 0.494 & 0.480 & 0.751 & 0.487 \\
\hline 23 & 0.742 & 0.238 & 0.764 & 0.244 & 0.491 & 0.503 & 0.756 & 0.497 \\
\hline 24 & 0.771 & 0.218 & 0.757 & 0.290 & 0.498 & 0.476 & 0.780 & 0.487 \\
\hline
\end{tabular}

then kept at this level for that particular subject throughout the remainder of the experiment. The signalto-noise ratios $\left(E / N_{O}\right)$ selected in this manner were approximately 9.1 .

The experimental subjects were Stanford University students who had been screened for normal hearing. They were paid at the rate of $\$ 2.50$ an hour. In addition they were told that a certain minimum level of performance would be required in order for them to continue in the experiment. Each daily run of 360 trials took approximately 45 minutes including a 10-minute rest period half way through the run.

In the main experiment, which began after the threeday calibration period, only two of the cue lights were used; these two appeared equally often, so that $\lambda_{1}=\lambda_{2}=1 / 2, \lambda_{3}=0$. Twelve subjects were each run through 360 trials a day for 24 days. During the first 9 days there was no cue-signal correlation, i.e., $\gamma_{1}=\gamma_{2}=1 / 2$. At the start of day 10 , unannounced to the subjects, a partial cue-signal correlation $\left(\gamma_{1}=3 / 4\right.$, $\gamma_{2}=1 / 4$ ) was introduced and maintained for the next ten days. Finally at the start of day 21 the subjects were returned unannounced to the original uncorrelated schedule. All randomizations in this experiment (and in Experiment II) were effected by randomly permuting a fixed number of events for each daily session; e.g., for each day in the first phase of Experiment I exactly 180 trials were $C_{1}$ and 90 of these were $S_{1}$. Thus the signal events $S_{1}$ and $S_{2}$ occurred equally often within each of the 24 daily sessions. However, during days 10 through 20 the $S_{1}$ signal occurred 75 per cent of the time when $c_{1}$ was presented and only 25 per cent of the time when $C_{2}$ was presented.

Results

The overall results of Experiment I are summarized in Table 1, which shows daily estimates of the relevant probabilities. These estimates are averages of the cor- responding statistics for individual subjects. The most pertinent feature of these data is the separation of the conditional probabilities of a correct response $\operatorname{Pr}\left(A_{i} \mid S_{i} C_{h}\right)$ effected by the introduction of a cue-signal correlation on day 10. Beginning with day 11 the estimates of $\operatorname{Pr}\left(A_{1} \mid S_{1} C_{1}\right)$ are consistently larger than those of $\operatorname{Pr}\left(A_{1} \mid S_{1} C_{2}\right)$ for each day of the cue dependent phase, while $\operatorname{Pr}\left(A_{2} \mid S_{2} C_{1}\right)$ is consistently smaller than $\hat{P}_{r}\left(S_{2} \mid S_{2} C_{2}\right)$. These bias effects appear to persist for at least two days after the return to an uncorrelated schedule. Taken as a whole these results indicate a significant conditional response bias effect controlled by the trial initiating cues. This interpretation is supported by an analysis of individual subject's performance over the last three days of the initial uncorrelated schedule, the correlated schedule, and the terminal uncorrelated schedule. The only statistically significant cue effects occurred in the correlated phase; here performances to either signal as a function of the cue event were significantly different ( $p<.05$ using a Mann Whitney $U$ Test).

Figure 1 presents daily estimates of the sensitivity

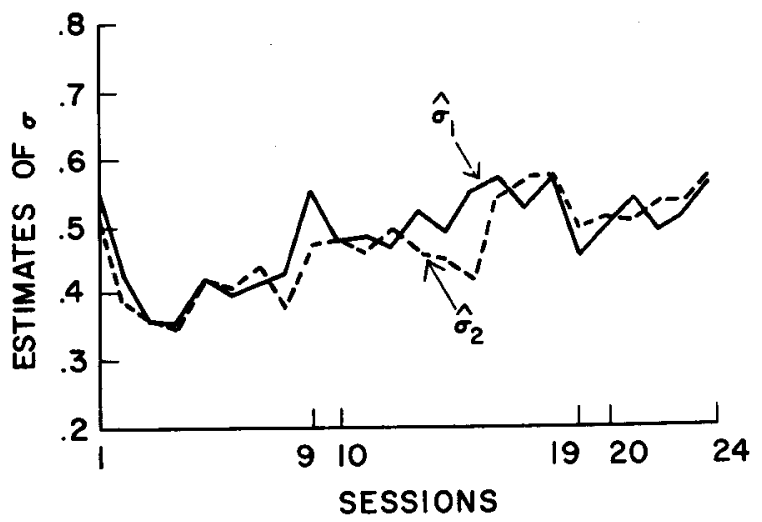

Fig. 1. Daily sensitivity estimates, $\hat{\sigma}_{1}$ and $\hat{\sigma}_{2}$, based on the data in Table 1. 


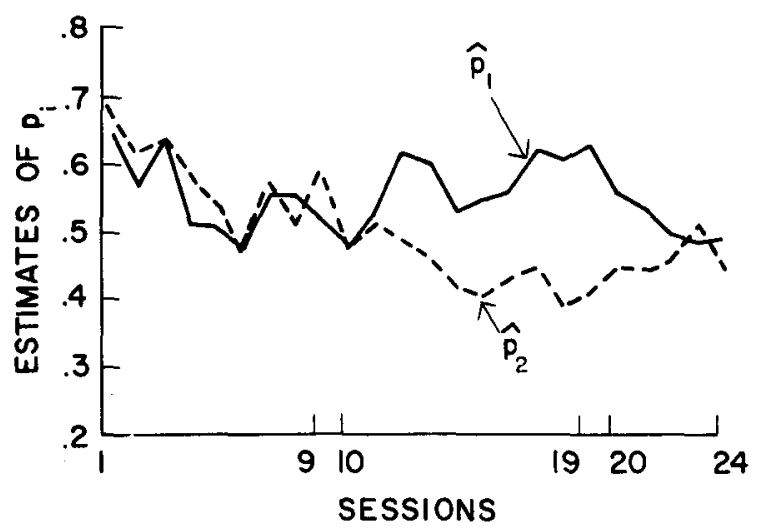

Fig. 2. Daily response bias estimates, $\hat{\mathbf{p}}_{1}$ and $\hat{\mathbf{p}}_{2}$, based on the data in Table 1.

parameter computed separately for $c_{1}$ and $c_{2}$ trials using the data in Table 1 and Eq. 3; these estimates are denoted $\hat{\sigma}_{1}$ and $\hat{\sigma}_{2}$ respectively. There does not appear to be any consistent difference in sensitivity on $c_{1}$ and $c_{2}$ trials. Figure 1 does suggest an initial decrease in sensitivity over days 1 through 4 , followed by a gradual, roughly monotonic, increase beginning on day 5 . These changes do not appear to be related to the cue-signal correlation.

Figure 2 shows daily estimates of the average values of the response bias parameters $p_{1}$ and $p_{2}$. These estimates were computed from the data in Table 1 using Eq. 4. Inspection of the figure indicates that a noncontingent bias in favor of response $A_{1}$ existed at the beginning of the experiment. This asymmetry was eliminated by the initial uncorrelated schedule, and with the introduction of a cue-signal correlation on day 10 the cue-contingent biases separate as predicted by the model. By day $19 \hat{\mathrm{p}}_{1}=.62$, while $\hat{\mathrm{p}}_{2}=.40$. Since the estimates of $p_{1}$ and $p_{2}$ in this phase are roughly symmetrical around $.5, \phi$ (in Eq. 6) should be around 1.0. In this case the asymptotes predicted for $p_{1}$ and $p_{2}$ are .75 and .25 , respectively. It is not clear from the graph whether the biases would have continued to increase toward these asymptotes if the correlated schedule had been continued beyond day 19. This question led to the design of Experiment II. In this second experiment subjects were given ample time to achieve an asymptotic response bias on each cue. (Latency data from Experiment I are reported in conjunction with the results of Experiment II.)

\section{Method}

\section{EXPERIMENT II}

The apparatus and the method of presenting the stimuli and recording responses were identical to those of Experiment I. However in Experiment II all three cues were employed with $\lambda_{1}=\lambda_{2}=\lambda_{3}=1 / 3$, and the same cue signal correlations obtained throughout the experiment: $\gamma_{1}=3 / 4, \gamma_{2}=1 / 2, \gamma_{3}=1 / 4$. Thus cues $c_{1}$ and $c_{3}$ were correlated with the signal events, whereas cue $C_{2}$ was uncorrelated. As in Experiment $I$ three preliminary sessions were spent in establishing a noise level for each subject such that he averaged close to 75 per cent correct responding. (During these preliminary sessions $C_{2}$ was the only cue employed.) Following the preliminary sessions ten subjects were each run for 360 trials a day for 20 consecutive days.

\section{Results}

The analysis of Experiment II focused on individual subject data. Table 2 presents estimates of the relevant conditional probabilities for each of the ten subjects. Each estimate is the corresponding mean proportion for a single subject over the last ten days of the experiment. Thus, for example, each estimate of $\operatorname{Pr}\left(A_{1}\right)$ is based on 3600 trials. For each subject these data indicate the predicted response bias effects as a function of the cues: in every case

$$
\hat{\operatorname{Pr}}_{\mathrm{A}}\left(A_{1} \mid S_{1} C_{1}\right)>\hat{\operatorname{Pr}}_{\mathrm{r}}\left(A_{1} \mid S_{1} C_{2}\right)>\hat{\operatorname{Pr}}_{\mathrm{r}}\left(A_{1} \mid S_{1} C_{3}\right)
$$

and

Table 2. Estimates of individual and average response probabilities based on the last ten days of Experiment II

\begin{tabular}{ccccccccc}
\hline Subject & $\hat{\operatorname{Pr}}\left(\mathrm{A}_{1} \mid \mathrm{S}_{1} \mathrm{C}_{1}\right)$ & $\hat{\operatorname{Pr}}\left(\mathrm{A}_{1} \mid \mathrm{S}_{1} \mathrm{C}_{2}\right)$ & $\hat{\operatorname{Pr}}\left(\mathrm{A}_{1} \mid \mathrm{S}_{1} \mathrm{C}_{3}\right)$ & $\hat{\operatorname{Pr}}\left(\mathrm{A}_{1} \mid \mathrm{S}_{2} \mathrm{C}_{1}\right)$ & $\hat{\operatorname{Pr}}\left(\mathrm{A}_{1} \mid \mathrm{S}_{2} \mathrm{C}_{2}\right)$ & $\hat{\operatorname{Pr}}\left(\mathrm{A}_{1} \mid \mathrm{S}_{2} \mathrm{C}_{3}\right)$ & $\hat{\operatorname{Pr}}$ (Correct) & $\hat{\operatorname{Pr}}_{r}\left(\mathrm{~A}_{1}\right)$ \\
\hline 1 & 0.768 & 0.708 & 0.677 & 0.327 & 0.263 & 0.237 & 0.736 & 0.497 \\
2 & 0.808 & 0.717 & 0.490 & 0.423 & 0.362 & 0.225 & 0.710 & 0.514 \\
3 & 0.891 & 0.624 & 0.450 & 0.520 & 0.163 & 0.092 & 0.771 & 0.458 \\
4 & 0.740 & 0.537 & 0.407 & 0.587 & 0.411 & 0.262 & 0.626 & 0.491 \\
5 & 0.890 & 0.615 & 0.371 & 0.712 & 0.346 & 0.130 & 0.707 & 0.506 \\
6 & 0.855 & 0.479 & 0.333 & 0.613 & 0.253 & 0.134 & 0.695 & 0.448 \\
7 & 0.881 & 0.587 & 0.307 & 0.730 & 0.432 & 0.141 & 0.676 & 0.512 \\
8 & 0.815 & 0.667 & 0.472 & 0.581 & 0.355 & 0.231 & 0.689 & 0.519 \\
9 & 0.860 & 0.429 & 0.389 & 0.773 & 0.311 & 0.253 & 0.639 & 0.498 \\
10 & 0.865 & 0.575 & 0.493 & 0.663 & 0.349 & 0.309 & 0.663 & 0.544 \\
Average & 0.837 & 0.594 & 0.439 & 0.593 & 0.324 & 0.202 & 0.691 & 0.499
\end{tabular}


Table 3. Three-cue study: Estimates of $\sigma$ and $\mathrm{Ph}_{h}$

\begin{tabular}{|c|c|c|c|c|c|c|}
\hline \multirow{2}{*}{ Subject } & \multicolumn{3}{|c|}{$\hat{o}_{h}=\hat{\operatorname{Pr}}\left(A_{1} \mid S_{1} C_{h}\right)-\hat{\operatorname{Pr}}\left(A_{1} \mid S_{2} C_{n}\right)$} & \multicolumn{3}{|c|}{$\hat{p}_{h}=\hat{\operatorname{Rr}}\left(A_{1} \mid S_{2} C_{h}\right) / 1-\hat{\sigma}_{h}$} \\
\hline & $\mathrm{c}_{1}$ & $c_{2}$ & $c_{3}$ & $\mathrm{c}_{1}$ & $\mathrm{c}_{2}$ & $\mathrm{C}_{3}$ \\
\hline 1 & 0.441 & 0.445 & 0.440 & 0.584 & 0.474 & 0.423 \\
\hline 2 & 0.384 & 0.355 & 0.265 & 0.687 & 0.561 & 0.306 \\
\hline 3 & 0.371 & 0.462 & 0.358 & 0.827 & 0.302 & 0.144 \\
\hline 4 & 0.153 & 0.127 & 0.144 & 0.693 & 0.470 & 0.306 \\
\hline 5 & 0.177 & 0.270 & 0.241 & 0.866 & 0.473 & 0.171 \\
\hline 6 & 0.242 & 0.226 & 0.200 & 0.809 & 0.327 & 0.167 \\
\hline 7 & 0.151 & 0.255 & 0.166 & 0.860 & 0.511 & 0.169 \\
\hline 8 & 0.233 & 0.312 & 0.241 & 0.758 & 0.516 & 0.304 \\
\hline 9 & 0.087 & 0.118 & 0.135 & 0.847 & 0.353 & 0.293 \\
\hline 10 & 0.204 & 0.226 & 0.184 & 0.834 & 0.451 & 0.379 \\
\hline Average & 0.244 & 0.270 & 0,237 & 0.776 & 0.444 & 0.266 \\
\hline
\end{tabular}

$$
\hat{\operatorname{Pr}}\left(A_{1} \mid S_{2} C_{1}\right)<\hat{\operatorname{Pr}}_{r}\left(A_{1} \mid S_{2} C_{2}\right)<\hat{\operatorname{Pr}}_{r}\left(A_{1} \mid S_{2} C_{3}\right)
$$

Table 2 also indicates that there was no apparent overall tendency to favor one response or the other independent of the cue and signal condition: the group mean of $\operatorname{Pr}\left(A_{1}\right)$ is .499 and individual subject values are all quite close to 5 .

Figure 3 shows ROC plots for each of the subjects together with the best fitting (by least squares) linear ROC curve having slope one. Inspection of the figure indicates that by and large the predicted ROC curves provide quite a good fit. These results support the assumption that sensitivity is independent of presentation schedules and cue conditions; deviations from such independence would produce either nonlinearity or linearity with a slope not equal to one.

Figure 3 reveals considerable individual differences in the spacing of points along the ROC curve. According to the model these differences must reflect differences in the conditional response biases of individual subjects. Table 3 shows group and individual subject estimates of the response biases $p_{1}, p_{2}$, and $p_{3}$. The estimates were computed using Eq. 3 and the data in Table 2. As would be expected from Fig. 3 the predicted ordering $\hat{p}_{1}>\hat{p}_{2}>\hat{p}_{3}$ is found in every case, and the group averages of $\hat{p}_{1}, \hat{p}_{2}$, and $\hat{p}_{3}$ are quite close to the predicted probability-matching values of $.75, .50$, and .25 . However the estimates for individual subjects reveal considerable variability; the $p_{1}$ estimates, for example, range from .866 to .584 . Although the predicted asymptotes in Eq. 6 depend on the parameter $\phi$, and thus allow for individual deviations from probability matching, it is not possible for the model of Eq. 5 to predict the patterns of deviation revealed by Table 3 . The $p_{h}$ estimates for subject 7 , for example, indicate biases which deviate from matching in the direction of optimal performance (i.e., in the direction of $p=1$ given $\gamma>1 / 2$, and $\mathrm{p}=0$ given $\gamma<1 / 2)$ for both $\gamma=.75$ and $\gamma=.25$, whereas Eq. 6 requires that if $p_{1}$ is greater than $\gamma_{1}, p_{3}$ must also be greater than $\gamma_{3}$ (cf. subject 10). Altogether four subjects $(2,5,6$, and 7$)$ show uniform deviations in the optimal direction, and three $(1,2$, and 4) show uniform deviations in direction of non-discriminative performance (i.e., in the direction of $p=.5$ for all cues). It is noteworthy, however, that in spite of the very large number of trials involved here no subject adopted a maximizing strategy.

Table 3 also shows group and individual subject
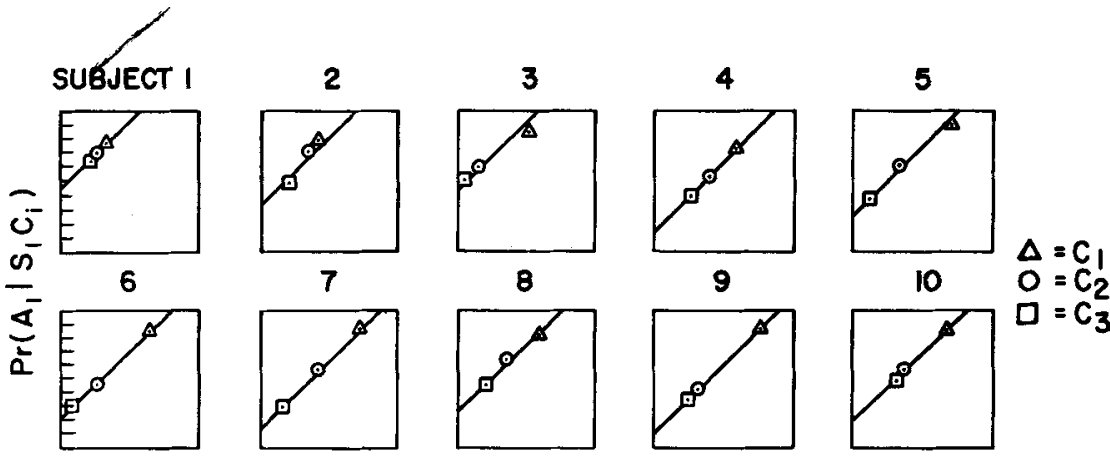

$\operatorname{Pr}\left(A_{1} \mid S_{2} C_{i}\right)$

Fig. 3. Receiver operating characteristics for individual subjects in Experiment II. 


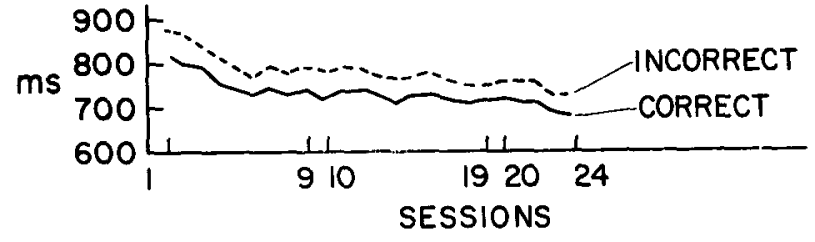

Fig. 4. Average response latencies for correct and incorrect responses during Experiment $\mathbf{I}$.

estimates of $\sigma$ computed separately for each cue condition. These estimates were computed from the data in Table 2 using Eq. 4. Although the average sensitivity estimates in Table 3 show $\hat{o}_{2}$ to be slightly greater than $\hat{\sigma}_{1}$ and $\hat{\sigma}_{3}$, there was no consistent ordinal relationship between sensitivity and cue condition for the individual subjects. A nonparametric test for such a relationship (Kruskal \& Wallis, 1952) was not significant.

\section{LATENCY RESULTS}

The large number of trials employed in Experiments I and II provided a unique opportunity to obtain good estimates of the latency distributions of individual subjects. In addition it was expected that the effects of the cue-signal correlations might be reflected in conditional response time distributions. This expectation was not confirmed. Analysis of the latency data from both experiments in terms of cue conditions failed to reveal any significant effects. The data did reveal a consistent $50 \mathrm{msec}$. difference in mean response times on correct and incorrect trials. Figure 4 shows this result for Experiment $I$. Analysis of individual subject latencies in Experiment II revealed a similar difference for each subject. These results are consistent with the detection model considered here if we assume that response times following an unambiguous sensory state $\left(s_{1}\right.$ or $\left.s_{2}\right)$ are realizations of a random variable $T_{u^{\prime}}$ while response times following any ambiguous sensory state $\left(b_{1}, b_{2}\right.$, or $\left.b_{3}\right)$ are realizations of another random variable $T_{\sigma}$. It is easily shown that these assumptions imply a constant difference between mean response times on correct and incorrect trials independent of cue condition (i.e., independent of response bias).
Examination of the individual subject latency distributions revealed rather consistent individual differences in the forms of these distributions. Figure 5 presents the individual subject distributions computed over successive four-day blocks. The right-hand column represents a pooling of responses over all subjects for each four-day block. Each of the histograms in the other columns represents 1440 responses by a single subjectall of his responses over the four sessions. The idiosyncratic nature of these distributions is quite striking; each subject's distribution maintains a consistent and unique form over at least the last four blocks of trials. Not surprisingly, in view of the considerable individual differences in the distributions, the group latency curve provides a very deceptive respresentation of the "typical" subject: compare the distributions for subject 6 , for example, to the average distribution. Discussion

The results of Experiments $I$ and II indicate that subjects in a signal detection task are able to discriminate several concurrent probability distributions over the signal events and to employ different response biases on a trial-by-trial basis as a function of the cues corresponding to these distributions. One implication of this finding is that a cued detection task can be used to simultaneously generate a number of points in the ROC space. An ROC curve generated in this fashion has the advantage of not being affected by session-to-session changes in sensitivity (cf. Fig. 1), since each sensitivity level is equally represented at each point of the curve.

The detection model considered here appears to give an adequate account of those aspects of the data that do not depend on the details of the response bias learning process. In addition, when a large number of trials were run on the correlated schedule the simple linear learning model accurately predicted average subject statistics, such as the mean response bias for each cue, and correctly ordered individual subject performances on each cue. However the model did not provide an adequate account of the details of individual performances. This is not surprising in view of the complex and quite idiosyncratic nature of the individual subject's performance in this task. In addition to the

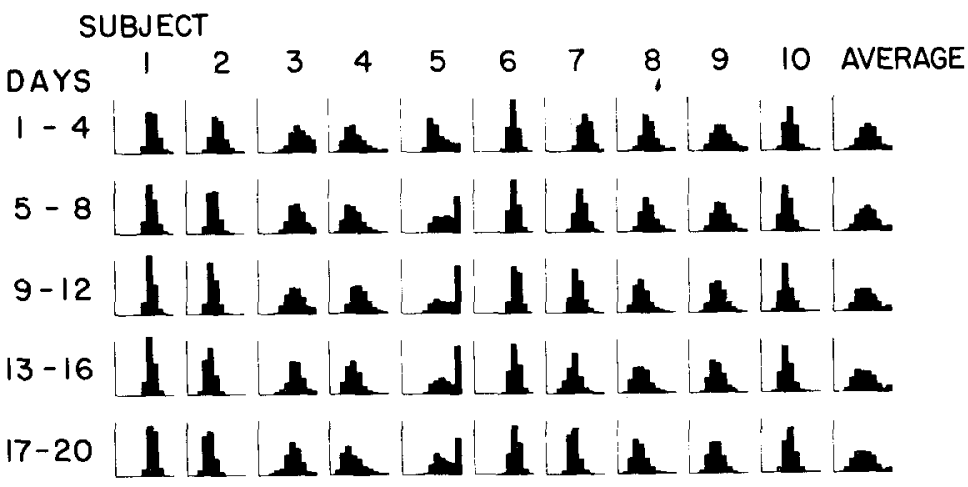

Fig. 5. Response latency histograms for individual subjects over successive blocks of four days during Experiment II. The horizontal axis is broken into 11 intervals. The 11 th (right-hand) interval represents all times greater than 1 sec.; the others represent latencies less than 1 sec. in . 1 sec. increments. 
variance in overall response biases observed in this experiment, a further analysis revealed a large amount of variability in the session-to-session performance of individual subjects. In view of these findings the success of the model in predicting average subject results should probably be attributed to its reflecting the gross features of a rather complex learning process. It is clear that in order to explain the bias learning process in detail a more complex learning model (which takes explicit account of the discrimination aspects of the situation) will be required.

\section{References}

Atkinson, R. C., Bogartz, w. H., \& Tumer, R. N. Supplementary report: Discrimination learning with probabilistic reinforcement schedules. J. exp. Psychol., 1959, 57, 349-350.

Atkinson, R. C., Bower, G. H., \& Crothers, E. J. An introduction to mathematical learning theory. New York: Wiley, 1965.
Atkinson, R. C., Carterette, E. C., \& Kinchla, R. A. Sequential phenomena in psychophysical judgments: a theoretícal analysis. Institute of Radio Engineers Transactions on Information Theory, 1962, IT8, S155-162.

Atkinson, R. C., \& Kinchla, R. A. A learning model for forcedchoice detection experiments. Brit. J. math. statist. Psychol., $1965,18,87-123$.

Kruskal, W. H., \& Wallis, W. A. Use of ranks in one-criterion variance analysis. $J$. Amer. Statist. Ass., 1952, 47, 583-621.

Luce, R. D. A threshold model for simple detection experiments. Psychol. Rev., 1963, 70, 61-79.

Popper, Juliet, \& Atkinson, R. C. Discrimination learning in a verbal conditioning situation. J. exp. Psychol., 1958, 56, 21-25.

Swets, J. A., Tanner, W. P., Jr., \& Birdsall, T. G. Decision processes in perception. Psychol. Rev., 1961, 68, 301-340.

\section{Nates}

1. Support for this research was provided by the National Aeronautics and Space Administration, Grant No. NGR-05-020-036. 2. The purpose of the alert signal was to eliminate a time-order error (favoring the second interval) that had been observed in earlier experiments. 